\title{
Effectiveness of mRNA BNT162b2 Vaccine 6 Months after Vaccination among Patients in Large Health Maintenance Organization, Israel
}

Jennifer Kertes, Sharon Baruch Gez, Yaki Saciuk, Lia Supino-Rosin, Naama Shamir Stein, Miri Mizrahi-Reuveni, Anat E. Zohar

Israel experienced a new wave of coronavirus disease during June 2021, six months after implementing a national vaccination campaign. We conducted 3 discrete analyses using data from a large health maintenance organization in Israel to determine whether IgG levels of fully vaccinated persons decrease over time, describe the relationship between IgG titer and subsequent PCRconfirmed infection, and compare PCR-confirmed infection rates by period of vaccination. Mean IgG levels steadily decreased over the 6-month period in the total tested population and in all age groups. An inverse relationship was found between $\lg G$ titer and subsequent PCR-positive infection. Persons vaccinated during the first 2 months of the campaign were more likely to become infected than those subsequently vaccinated. The vaccinated group $\geq 60$ years of age had lower initial IgG levels and were at greater risk for infection. The findings support the decision to add a booster vaccine for persons $\geq 60$ years of age.

Coronavirus disease (COVID-19) was identified in Israel at the end of February 2019 (1). As in other countries, Israel has experienced several infection waves. The third wave, largely attributed to entry of the Alpha virus variant into Israel, began during in September 2020; at its peak, >8,000 new cases were being identified daily (2). Israel was among the first countries to introduce a national vaccination campaign using the mRNA BNT162b2 vaccine (Pfizer-BioNTech, https://www.pfizer. com). The BNT162b2 vaccine received emergency approval for use by the US Food and Drug Administration after the vaccine showed $95 \%$ efficacy over an average 2 -month follow-up period $(3,4)$. The vaccine was initially approved for any person $\geq 16$

Author affiliation: Maccabi HealthCare Services, Tel Aviv-Jaffa Israel

DOI: https://doi.org/10.3201/eid2802.211834 years of age, with a recommended 21-day interval, 2-dose administration.

The vaccine campaign began on December 20, 2020 (concurrent with a 2-month nationwide lockdown), first targeting all healthcare workers and the population $\geq 60$ years of age and quickly extending to all persons $\geq 16$ years of age. Initially, those persons who had a previous infection were not eligible for vaccination, but within 3 months, policy was changed to offer a single dose to all persons who had a previous infection. By April 2021, $>50 \%$ of persons $\geq 16$ years of age and $88 \%$ of persons $\geq 50$ years of age countrywide had been fully vaccinated (2). The number of new cases decreased to 140 cases/day by April 2021 (2). Initial population-based studies in Israel comparing vaccinated and unvaccinated groups reported vaccine effectiveness rates of $95 \%(5,6)$.

One of the biggest questions regarding the vaccine is the length of protection provided. In publishing third-phase research results, Pfizer-BioNTech reported a 91\% efficacy rate over a 6-month follow-up period and an estimated $6 \%$ decrease in efficacy every 2 months (7). Population-based observational studies in Israel are no longer a feasible method of evaluating long-term effectiveness of the vaccine, given that most persons have now been fully vaccinated. Infection rates in Israel increased again during June-September 2021 (fourth wave), and most (97\%) positive cases were infected with the Delta variant (B.1.617.2) (G. Rahib, Israel Ministry of Health Laboratories, pers. comm., 2021 Aug 8). Initial serologic studies of the Delta variant suggest that the BNT162b2 vaccine provides protection against Delta variant infection, but at lower rates than for the Alpha variant (88\% vs. 93.7\%) (8). Given the increase in infection rates, the dilemma arose whether this increase was attributable to 
reduced effectiveness of the vaccine against the Delta variant or a waning of protection provided by the vaccine over time.

The objective of this study was to determine if the BNT162b2 vaccine had become less effective in preventing infection, and if so, in which population groups and to what degree. To meet this objective, we conducted 3 discrete analyses to answer the following questions. First, do antibody levels (IgG) of those fully vaccinated decrease over time and if so, for who and how quickly? Second, what is the relationship between antibody level (IgG) and subsequent PCR-confirmed infection? Third, is there a difference in PCRconfirmed infection incidence rates between persons vaccinated in the initial months of the vaccination campaign and persons vaccinated later?

\section{Methods}

We conducted a series of retrospective cohort analyses to meet the study objectives. We extracted all data from the Maccabi Healthcare Services database (https:/ / www.maccabi4u.co.il/1781-he/Maccabi. aspx). Maccabi is the second-largest health maintenance organization (HMO) in Israel and provides healthcare coverage for $>2.5$ million citizens $(27 \%$ of the population of Israel). The database includes demographic data (date of birth, sex, socioeconomic status based on census, and national survey classifications applied to home address); laboratory data (all PCR and IgG test results); and health status data (chronic illness registries, such as heart disease, hypertension, chronic kidney disease [CKD], diabetes and immunosuppressive disorder, based on hospital and community-based diagnoses and procedures, and relevant laboratory and test results). The study was approved by the Maccabi Helsinki Committee (\#0178-20-MHS). Informed consent was waived because all data extracted from the database were anonymized and aggregated.

\section{Testing Procedures}

PCR testing is conducted free of charge for any HMO member who has symptoms or reported exposure to a confirmed case. Testing is conducted by using real-time reverse transcription PCR (Allplex 2019-nCoV Assay; Seegene Inc., https://www. seegene.com). We offered serologic testing to specific target populations, such as employees (19\%) and residents and employees of geriatric medical and retirement home facilities owned by the HMO $(4 \%)$ at discrete points in time, but most $(77 \%)$ testing was carried out in the general HMO population for whom testing is freely available upon request (patients initiative). We conducted IgG testing by using severe acute respiratory syndrome coronavirus 2 (SARS-CoV-2) spike-specific antibodies and a follow-up chemo-luminescence immunoassay (Quant II IgG anti-Spike CoV2-SARS; Abbott Laboratories, https://www.abbott.com) and reported as arbitrary units per milliliter (AU/mL). Antibody levels are reported numerically, except for outliers $(<21 \mathrm{AU} / \mathrm{mL}$ and $>40,000 \mathrm{AU} / \mathrm{mL})$, which are coded. Coded results were converted to numeric results ( $<21$ to 21 and $>40,000$ to 40,001$)$.

\section{IgG Levels of Vaccinated Population over Time}

All HMO members who had received both vaccine doses and had a subsequent IgG test for SARS-CoV-2 antibodies $\geq 7$ days after the second vaccination were included in this component of the study. The study period extended from January 11, 2021 (when those first vaccinated reached day 7 after the second dose), through July 7, 2021. We mapped IgG results over a 180-day period by using demographic and health characteristics (age group, sex, socioeconomic status, and presence of selected chronic illnesses).

\section{Relationship between IgG Levels and Subsequent SARS-CoV-2 Infection}

We included all HMO members who had a PCR test (irrespective of vaccination status) during June 1-July 14, 2021 (peak of fourth wave of infection), and an IgG serologic test 7-120 days before the PCR test in this component of the study. We used the most recent test result for persons who had $>1$ test. We used the most recent PCR test date if all results were negative and the date of the first positive PCR result for persons who had $>1$ result. We calculated the proportion of participants who had subsequent positive PCR results by antibody level status.

\section{Comparison of Infection Rates by Vaccination Period}

We included all HMO members who as of June 9, 2021 , were $\geq 7$ days post-second vaccination dose and had no previous positive PCR result in this component of the study. We excluded from analysis members who received 3 doses or had an appointment to receive the third dose $(n=320)$ during the follow-up period. (At this time, a recommendation to offer a booster vaccination for persons who had an immune-suppressive disorder had been authorized.) We categorized the study population by using vaccination completion: January-February 2021 and March-May 2021. For both groups, we calculated the proportion who were PCR positive during June 9-July 18, 2021 (yes/no). 


\section{Statistical Analyses}

We used Mann-Whitney and Kruskal-Wallis tests to compare antibody levels over time between different population groups. We used linear regression to identify those factors associated with serologic levels. Natural logarithm (ln) of serologic levels showed a normal distribution and was selected as the outcome variable. Other variables we entered into a hierarchical model were days from vaccination; age, sex, and socioeconomic status; and selected chronic illnesses.

We used $\chi^{2}$ analyses to test the association between serologic levels (categorized) and PCR outcomes. We categorized serologic status into $\leq 300 \mathrm{AU} / \mathrm{mL}$ or $>300 \mathrm{AU} / \mathrm{mL}$ We calculated Kaplan-Meier survival curves to compare time from serologic test to positive PCR result for the serologic categories by using log-rank tests. We defined an event as a positive PCR result. Time to event was the number of days from a serologic test to PCR, with censoring for those who died, left the HMO, or had a follow-up period of $<120$ days. We used logistic regression analysis to compare PCR-positive outcomes between vaccination periods, while controlling for age group, socioeconomic status, and presence of chronic illness (heart disease, hypertension, diabetes, CKD, and immunosuppressive disorder).We performed statistical analyses by using SPSS Statistics 25 (https:/ / www.ibm.com) and R version 3.6.2 (https:/ / cran.r-project.org).

\section{Results}

\section{IgG Levels of Vaccinated Population Over Time}

The study population consisted of 8,395 persons (Table 1). Of all HMO members who received both vaccine doses, those subsequently tested for IgG were more likely to be male, younger (18-44 years of age), and in a higher socioeconomic bracket and less likely to have a chronic illness than those not tested for IgG.

We found that serologic levels in the study population decreased over time, from a mean of 14,008 for those tested within a month of being vaccinated to a mean of 1,411 for those tested in the sixth month after vaccination (Table 2). We observed a decrease over time in all subpopulation groups when results were stratified by age group, sex, socioeconomic status, and selected chronic illnesses (Table 2). The largest mean differences between subpopulations were observed in their initial serologic levels (within the first month). Mean serologic levels for participants $\geq 60$ years of age $(n=1,004$, mean $9,433)$ were approximately half of those for participants $<60$ years of age $(n=1,453$, mean 17,169$)$ in the first month, attenuating to a $<10 \%$ difference 6 months later (Figure 1). Large differences in initial serologic levels were also observed for participants with chronic illness, in particular participants with an immunosuppressive disorder, CKD or heart

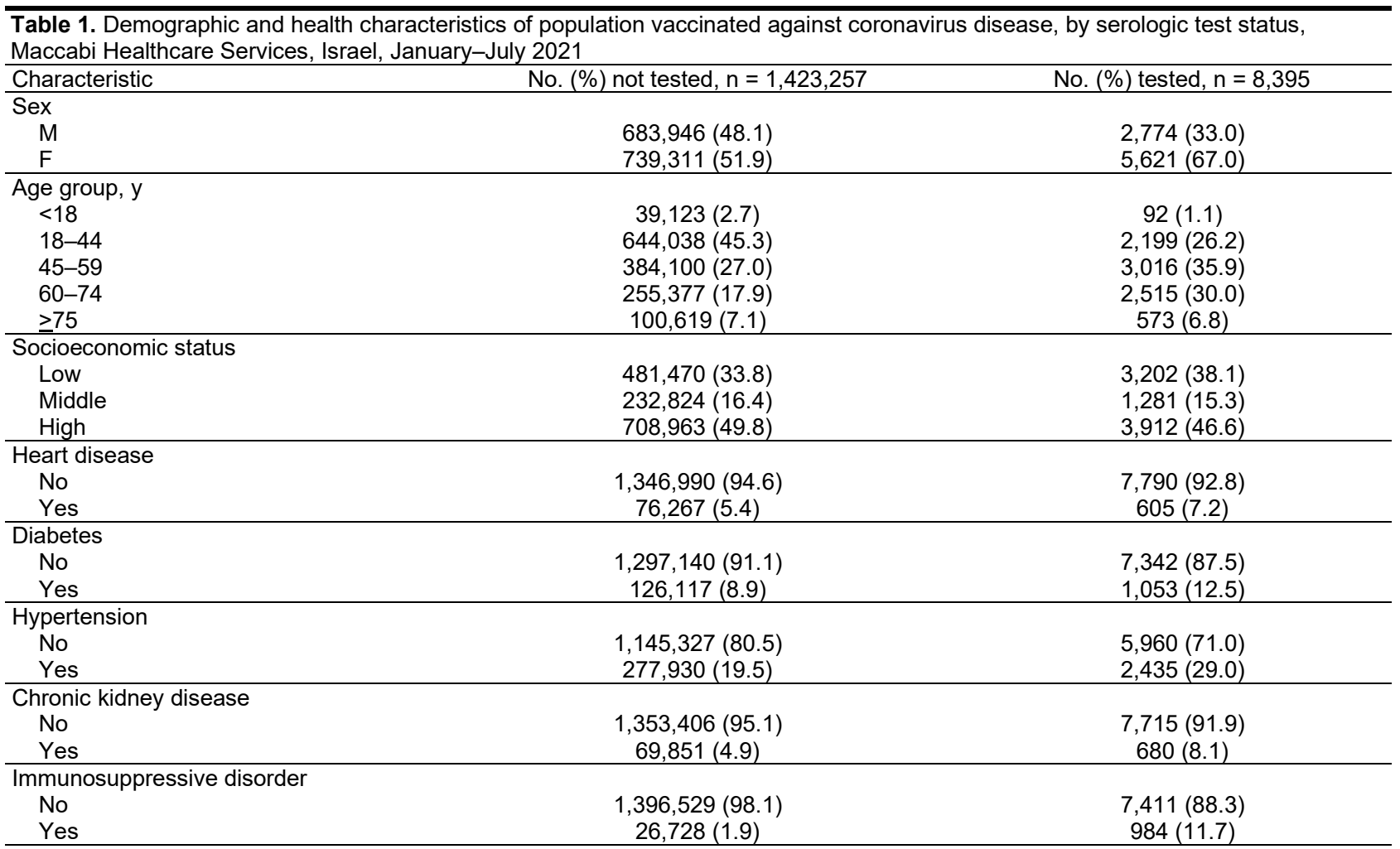


Table 2. Mean antibody level by demographic and health variables and time from vaccination against coronavirus disease for population vaccinated against coronavirus disease, Maccabi Healthcare Services, Israel, January-July 2021

\begin{tabular}{|c|c|c|c|c|c|c|c|}
\hline \multirow[b]{2}{*}{ Characteristic } & \multicolumn{6}{|c|}{ Days from vaccination to serologic test } & \multirow[b]{2}{*}{$\mathrm{p}$ value } \\
\hline & $7-29$ & $30-59$ & $60-89$ & $90-119$ & $120-150$ & $>150$ & \\
\hline Total population & 2,457 & 1,845 & 946 & 827 & 500 & 1,820 & \\
\hline \multicolumn{8}{|l|}{ Antibody levels } \\
\hline Mean & 14,008 & 8,175 & 4,365 & 2,706 & 1,773 & 1,411 & $<0.001$ \\
\hline SD & 12,146 & 7,742 & 5,022 & 3,957 & 1,934 & 1,751 & \\
\hline Median & 11,322 & 6,080 & 2,974 & 1,683 & 1,217 & 1,217 & \\
\hline \multicolumn{8}{|l|}{ Sex } \\
\hline No. male & 1,075 & 676 & 354 & 269 & 105 & 295 & \\
\hline Mean antibody level & 12,278 & 6,837 & 3,799 & 2,633 & 1,695 & 1,309 & $<0.001$ \\
\hline No. female & 1,382 & 1,169 & 592 & 558 & 395 & 1,525 & \\
\hline Mean antibody level & 15,354 & 8,949 & 4,703 & 2,740 & 1,794 & 1,431 & \\
\hline \multicolumn{8}{|l|}{ Age group, $y$} \\
\hline No. $<18$ & 32 & 40 & 14 & 6 & 0 & 0 & \\
\hline Mean antibody level & 29,781 & 15,348 & 9,971 & 9,421 & & & $<0.001$ \\
\hline No. $18-44$ & 677 & 469 & 262 & 201 & 142 & 448 & \\
\hline Mean antibody level & 18,522 & 9,866 & 5,621 & 3,271 & 2,006 & 1,479 & \\
\hline No. 45-59 & 744 & 602 & 288 & 237 & 224 & 921 & \\
\hline Mean antibody level & 15,396 & 8,875 & 4,279 & 2,793 & 1,929 & 1,419 & \\
\hline No. $60-74$ & 821 & 599 & 302 & 264 & 99 & 430 & \\
\hline Mean antibody level & 9,999 & 6,280 & 3,684 & 2,670 & 1,478 & 1,256 & \\
\hline No. $\geq 75$ & 183 & 135 & 80 & 119 & 35 & 20 & \\
\hline Mean antibody level & 6,892 & 5,468 & 2,147 & 1,316 & 668 & 912 & \\
\hline \multicolumn{8}{|l|}{ Socioeconomic status } \\
\hline No. low & 553 & 278 & 143 & 113 & 59 & 135 & \\
\hline Mean antibody level & 15,994 & 10,048 & 4,481 & 4,056 & 2,443 & 1,625 & $<0.001$ \\
\hline No. middle & 1,088 & 827 & 414 & 392 & 259 & 932 & \\
\hline Mean antibody level & 13,989 & 8,473 & 4,739 & 2,523 & 1,695 & 1,468 & $<0.001$ \\
\hline No. H\high & 816 & 740 & 389 & 322 & 182 & 753 & \\
\hline Mean antibody level & 12,687 & 7,139 & 3,924 & 2,454 & 1,667 & 1,301 & $<0.001$ \\
\hline \multicolumn{8}{|l|}{ Underlying conditions } \\
\hline \multicolumn{8}{|l|}{ Heart disease } \\
\hline No. patients & 206 & 165 & 79 & 75 & 25 & 55 & \\
\hline Mean antibody level & 7,341 & 4,307 & 2,520 & 2,455 & 690 & 1,575 & $<0.001$ \\
\hline \multicolumn{8}{|l|}{ Diabetes } \\
\hline No. patients & 377 & 245 & 121 & 123 & 57 & 130 & \\
\hline Mean antibody level & 8,624 & 6,647 & 2,742 & 2,189 & 843 & 1,401 & $<0.001$ \\
\hline \multicolumn{8}{|l|}{ Hypertension } \\
\hline No. patients & 803 & 572 & 274 & 290 & 133 & 363 & \\
\hline Mean antibody level & 9,930 & 6,624 & 3,032 & 2,118 & 1,341 & 1,409 & $<0.001$ \\
\hline \multicolumn{8}{|l|}{ Chronic kidney disease } \\
\hline No. patients & 248 & 163 & 81 & 88 & 45 & 55 & \\
\hline Mean antibody level & 6,756 & 4,331 & 2,614 & 2,339 & 887 & 1,910 & $<0.001$ \\
\hline \multicolumn{8}{|l|}{ Immunosuppressive disorder } \\
\hline No. patients & 307 & 280 & 156 & 126 & 57 & 58 & \\
\hline Mean antibody level & 6,824 & 4,371 & 2,336 & 1,500 & 1,033 & 1,813 & $<0.001$ \\
\hline
\end{tabular}

disease. Initial (first month) serologic levels increased by socioeconomic level.

Of all persons who were vaccinated with both doses, $2.8 \%$ also had a positive PCR result. Comparable decreases in serologic means by month were observed in this group, as in others. However, the mean serologic level for those tested in the first 7-30 days was much higher than that for the total study population $(22,630 \mathrm{AU} / \mathrm{mL}$; $\mathrm{p}<0.001)$.

When demographic and health variables were entered into a linear regression model (Appendix Table 1, https://wwwnc.cdc.gov/EID/article/27/2/211834-App1.pdf), all factors remained independently associated with serologic levels; the highest coefficient was observed for participants who had an im- munosuppressive disorder. No multicollinearity was observed between the factors in the regression model.

\section{Relationship between IgG Levels and Subsequent SARS-CoV-2 Infection}

Demographic and health characteristics of HMO members who had a serologic result and who had subsequently been tested by PCR were similar to those who had no PCR test result (Table 3), with the exception of socioeconomic status and diabetes status. Persons who were tested by PCR were more likely to be in the lower socioeconomic bracket and have diabetes than persons not tested by PCR.

Of persons who had both serologic and PCR tests $(\mathrm{n}=5,141), 57 \%$ had a serologic test result of $\leq 150 \mathrm{AU} /$ 


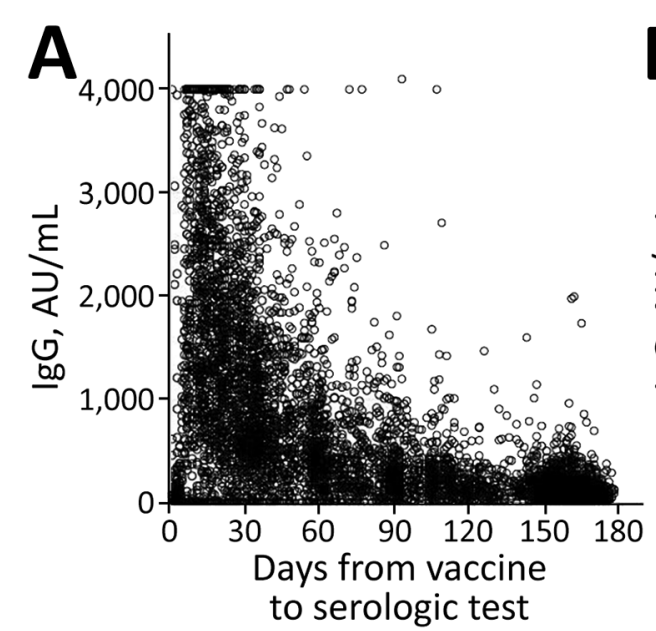

$\mathrm{mL}, 6 \%$ had a result of $150-299 \mathrm{AU} / \mathrm{mL}, 10 \%$ had a result of 300-799 AU/mL, and $27 \%$ had a result $>800 \mathrm{AU} /$ $\mathrm{mL}$. The proportion of participants with a positive PCR result were $1.2 \%$ for those who had serologic levels $\leq 150$ $\mathrm{AU} / \mathrm{mL}, 1.3 \%$ for those who had serologic levels of 150 $299 \mathrm{AU} / \mathrm{mL}, 0.2 \%$ for those who had serologic levels of 300-799 AU/mL, and 27\% for those who had serologic levels of $\geq 800 \mathrm{AU} / \mathrm{mL}$ ( $p=0.004$. Mean serologic levels for the 42 study participants who had a positive PCR result were $175 \mathrm{AU} / \mathrm{mL}(\mathrm{SD} \pm 490 \mathrm{AU} / \mathrm{mL}$ ) compared a mean serologic level of 2,057 AU/mL (SD $\pm 6,030 \mathrm{AU} /$ $\mathrm{mL})$ for those with a negative result $(\mathrm{p}<0.001)$.
Figure 1. IgG levels for population vaccinated with mRNA BNT162b2 vaccine (Pfizer-BioNTech, https://www. pfizer.com) against coronavirus disease over time, by age group, Maccabi Healthcare Services, Israel, January-June 2021. A) $<60$ years of age. B) $\geq 60$ years of age.

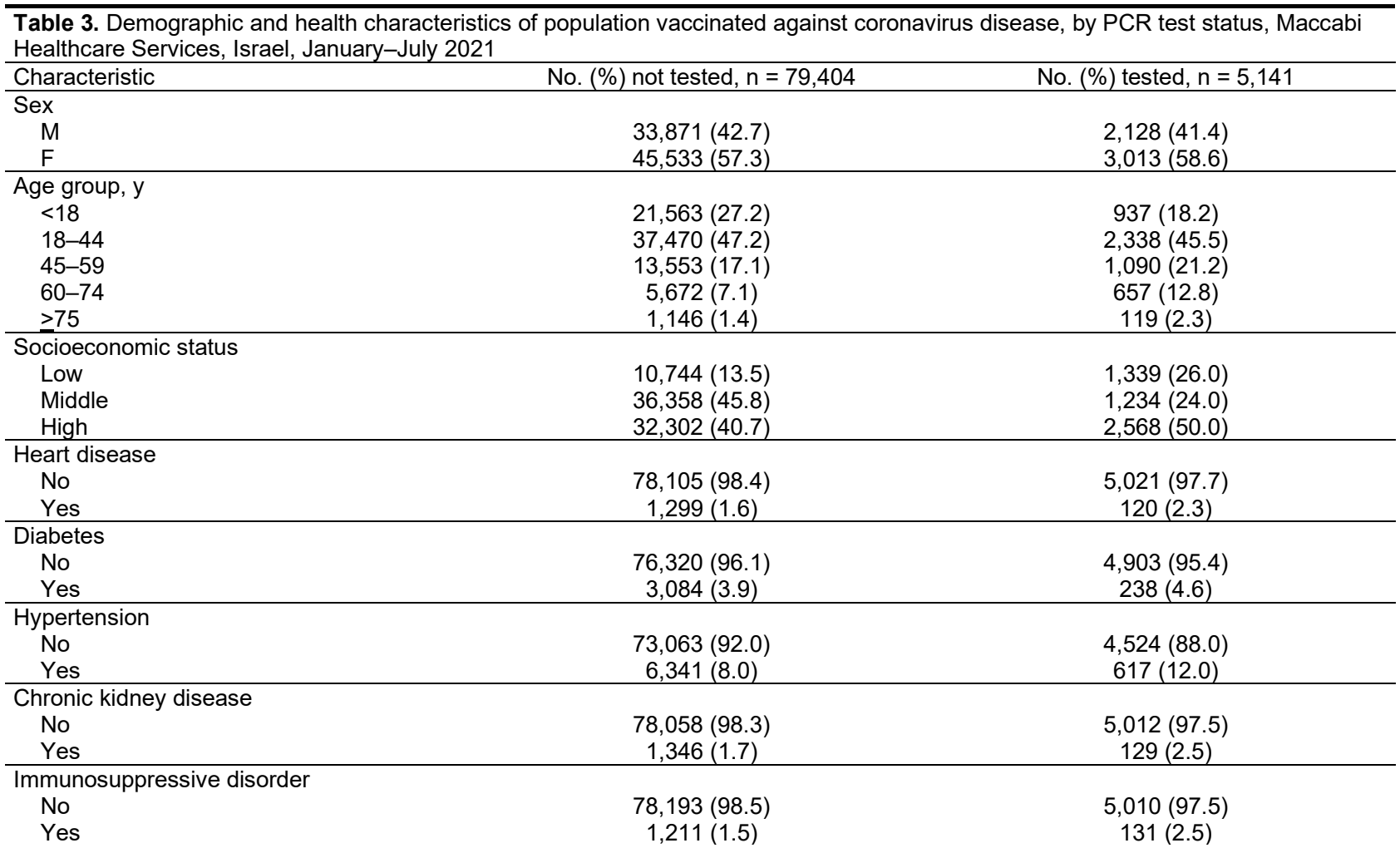




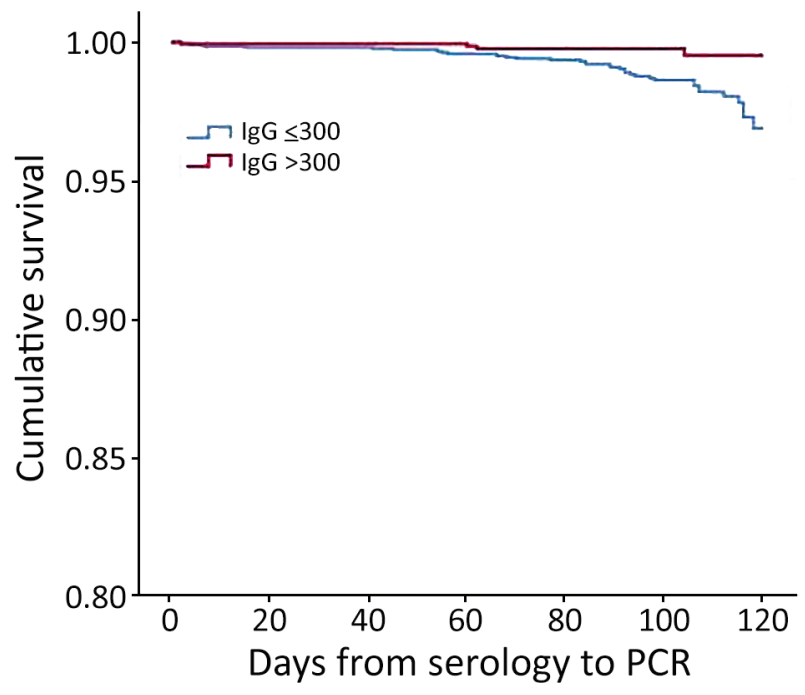

Figure 2. Kaplan-Meier cumulative survival for PCR-positive outcome for population vaccinated with mRNA BNT162b2 vaccine (Pfizer-BioNTech, https://www.pfizer.com) against coronavirus, by antibody (IgG) level, Maccabi Healthcare Services, Israel, JuneJuly 2021.

survival rates than participants who had higher serologic levels $( \pm 300 \mathrm{AU} / \mathrm{mL} ; \mathrm{p}=0.03$ by log-rank test).

\section{Comparison of Infection Rates by Vaccination Period}

At the time of the study, $86 \%$ of those eligible for vaccination ( $\geq 16$ years of age; $n=1,423,098$ ) had received both doses in the HMO $(90 \%$ of those $\geq 60$ years of age). We compared demographic and health variables between those who were vaccinated in the first 2 months with those vaccinated later (Table 4). Those who were vaccinated in the first 2 months were more likely to be older, in a higher socioeconomic bracket, and have higher rates of chronic illness. We found that $1,518(0.19 \%)$ of those vaccinated during January-February 2021 were PCR positive compared with $644(0.11 \%)$ of those vaccinated during March-May $2021(\mathrm{p}<0.001)$. Univariate analyses (Appendix Table 2) also showed that age, sex, socioeconomic status and presence of chronic illnesses (health disease, diabetes, hypertension, and CKD) were associated with having a positive PCR result.

Factors associated with subsequent infection (positive PCR result) in a logistic regression model (Table 5) were socioeconomic status, age group, vaccination period, sex, and heart disease. When controlling for all other factors, we found that members vaccinated first were 1.6 times more likely to get infected with COVID-19 than those vaccinated later.

\section{Discussion}

In this study, we found that IgG serologic levels for SARS-CoV-2 virus decreased progressively over time for the total vaccinated population and in each subpopulation when stratified by demographic and

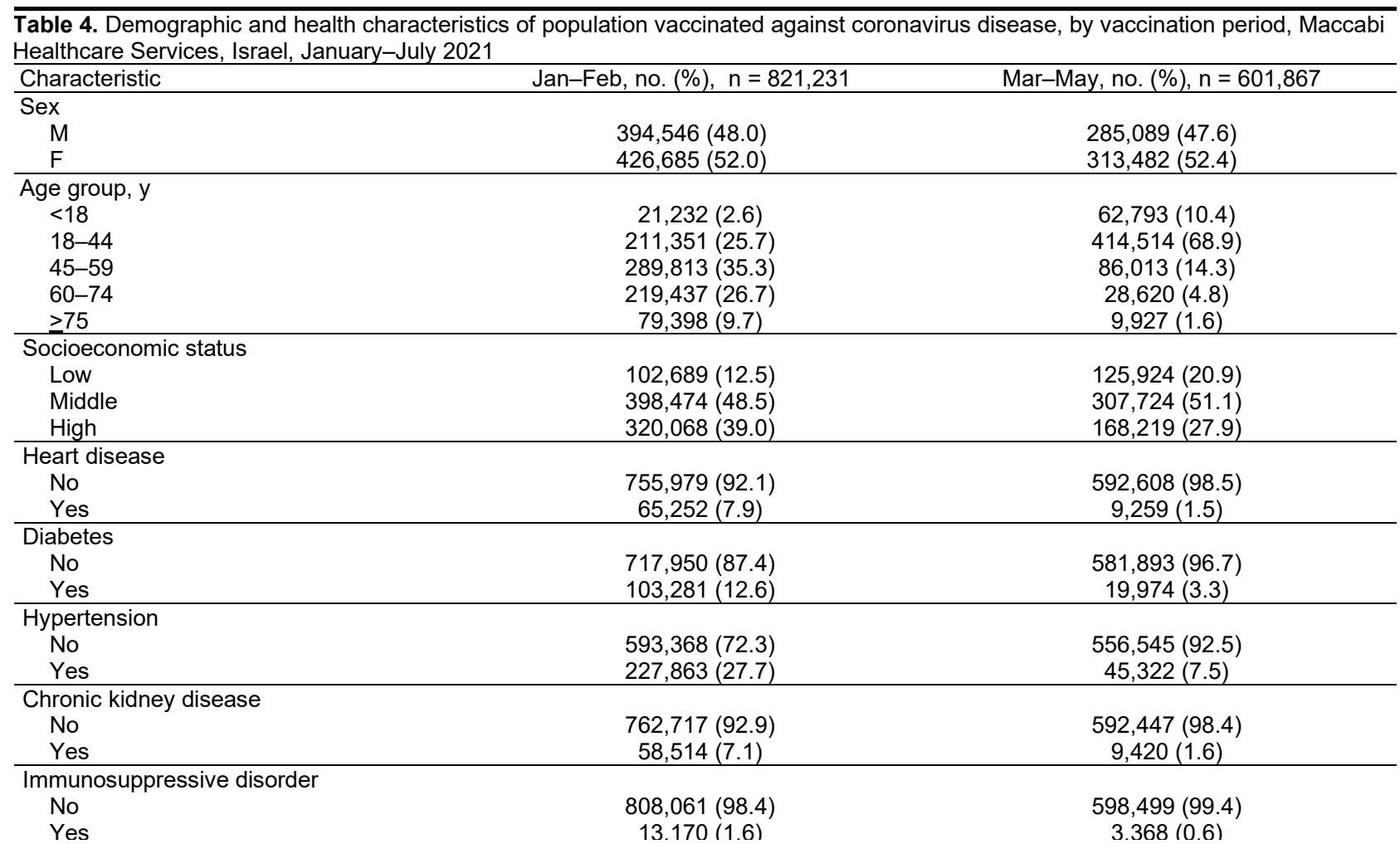


Table 5. Factors associated with coronavirus disease for population vaccinated against coronavirus disease, by logistic regression model, Maccabi Healthcare Services, Israel, January-July 2021

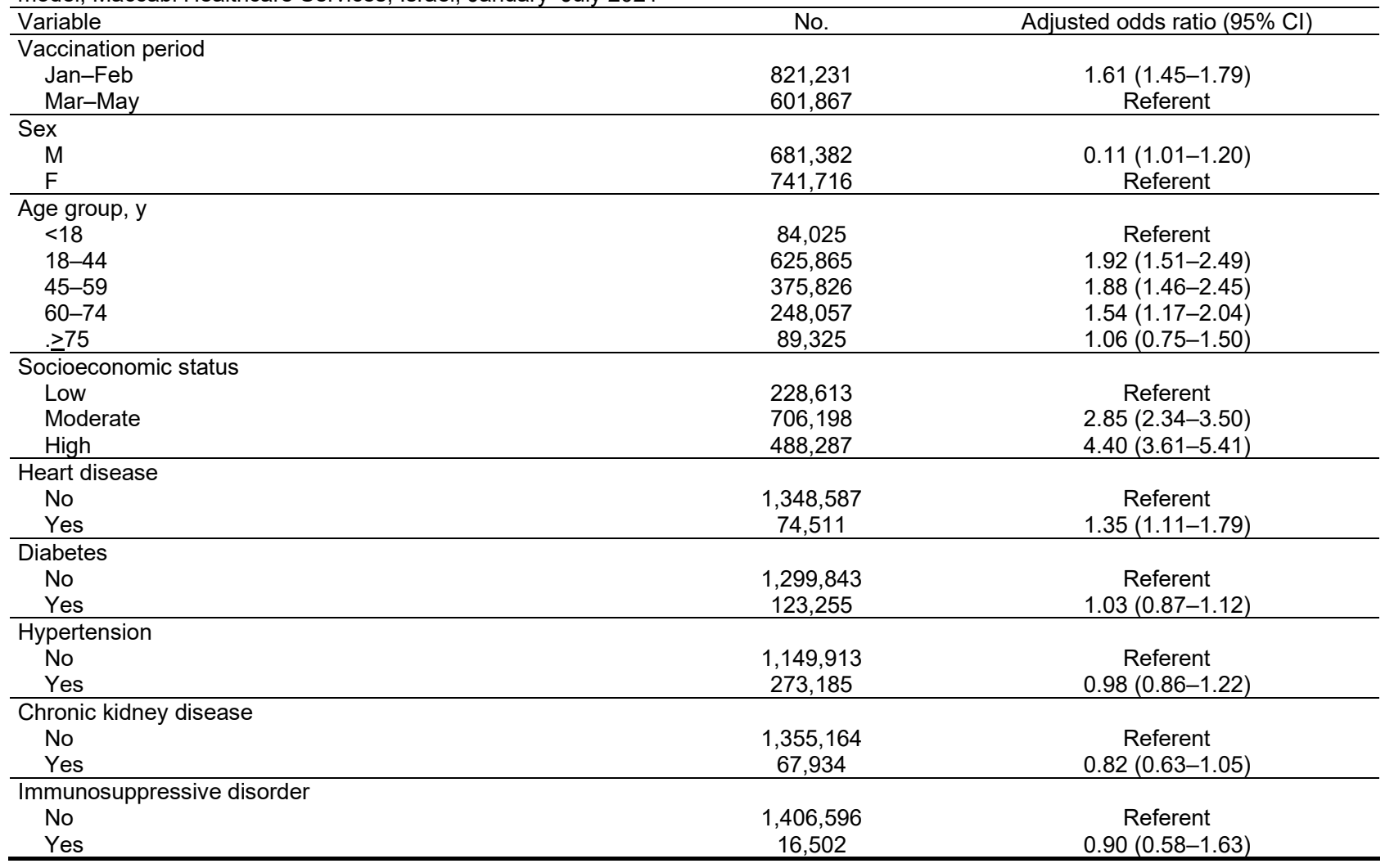

health variables. We also found an association between serologic levels and subsequent risk for infection, wherein participants who had a serologic level $\leq 300 \mathrm{AU} / \mathrm{mL}$ were more likely to get COVID-19 than those who had a serologic level $>300 \mathrm{AU} / \mathrm{mL}$. We established that those vaccinated at the beginning of the national vaccination campaign were more likely to get infected (during the current wave of infection) than those vaccinated later. These findings suggest that effectiveness of the vaccine decreases over time and that the current wave of infection can be attributed, at least in part, to the reduced effectiveness of the vaccine over time.

Initial serologic studies focused on patients found to be PCR positive for COVID-19 reported a decrease over time of antibody presence from time of infection (9-11). Fewer studies have looked specifically at serologic response of the vaccinated population. Most of the studies based on vaccinated populations reported $\approx 100 \%$ seroconversion rates but had short follow-up periods $(12,13)$. Serologic levels were much higher among the vaccinated population than those convalescing after infection (12) and among those $<50$ years of age ((13). In a case-control study of PCR-positive case-patients divided by previous vaccination status (yes/no), Lopez-Bernal et al. (8) found that those vaccinated (2 doses) with the BNT162b2 vaccine and were infected with the Alpha variant achieved $93.7 \%$ vaccine effectiveness rate compared with an $88 \%$ vaccine effectiveness rate for those infected with the Delta variant.

We did not find published studies that described serologic status over longer follow-up periods for a vaccinated population. Mean levels of IgG decreased progressively over time for all subpopulations in this study. The difference between the groups was mostly evident in initial (first month) starting means; the elderly and those having chronic illness had lower levels, but these levels attenuated to more comparable levels between groups 6 months after vaccination. In a large household study in the United Kingdom (14), IgG response measured over the first 3 months after vaccination found higher seroconversion rates for younger age groups (20-40 years of age), female participants, those receiving both doses, vaccination with BNT162b2 vaccine compared with AstraZeneca (https:/ / www.astrazeneca.com) vaccine, and those with evidence of a previous infection. Low responders were older and had higher prevalence of chronic illness/disease, such as patients receiving immune suppressants or who had diabetes. These 
same population groups were found in this study to start with lower serologic levels and have lower mean serologic levels 6 months after vaccination.

One of the many unknowns regarding COVID-19 is to what extent IgG is indicative of protection against the virus. The manufacturer's recommended cutoff indicating a positive serologic response $(<50 \mathrm{AU} / \mathrm{mL})$ is much lower than the mean serologic levels we found at 6 months after vaccination. Are higher levels indicative of higher protection? Other mechanisms of protection, such as antiviral $\mathrm{T}$ and $\mathrm{B}$ cell memory, have been suggested as offering protection, even in the absence of seroconversion (15). In a meta-analysis, Khoury et al. (16) found a strong relationship between mean neutralization levels and reported protection. They further estimated that protection was likely to occur over 250 days, although with still largely preserved protection from severe infection. In the second component of our study, we found an association between serologic level and PCR outcome in which increased serologic level was associated with decreasing infection rates. Using a cutoff value of $300 \mathrm{AU} / \mathrm{mL}$, we found higher rates of infection for those with low serologic levels. However, given that those coming for testing were not randomly selected, repeat studies in a large randomly selected population are required to confirm this cutoff value.

Few data are available to compare vaccine effectiveness over time, and observational follow-up studies are becoming less appropriate, given the potential bias between those electing to vaccinate and those who do not. Pfizer-BioNTech published a recent efficacy study that compared symptomatic infection rates between vaccinated and unvaccinated groups over a period of 6 months (7). Vaccine efficacy for infection decreased from $96 \%$ within the first 2 months post-second dose to $84 \%$ vaccine efficacy $4-6$ months post-second dose. Consistent with the findings of Pfizer-BionTech, we found higher rates of COVID-19 infection among those vaccinated in the initial months of the vaccine campaign compared with those vaccinated later. Even after controlling for age (those vaccinated first were more likely to be older), incidence rates were higher in the first vaccinated group. Were most of the fourth wave of infections attributable to the Delta virus, we would have expected consistent incidence rates, irrespective of when the individual was vaccinated. We suggest that the difference found here between time periods indicates a reduction in vaccine effectiveness over time. However, we cannot rule out some contribution of the Delta variant to reduced effectiveness.
One limitation of our study was that test findings were not based on repeated tests in the same population but a description of the results over time of those coming for a serologic test. Those coming for serologic and PCR testing were not randomly selected groups but, rather, persons volunteering in a study or, more commonly, requesting to be tested. Participants requesting a test (serologic and PCR) might have had greater concerns regarding exposure, infection, or perceived infection risk, potentially increasing the proportion of persons who had lower serologic levels or a positive PCR result. We calculated mean serologic levels for each subpopulation, despite the potential for outlier measures to skew results, to enable statistical comparison between subpopulation groups. Numbers were small for some stratified data, particularly for the 120-149 day period, and should be interpreted with caution. Study findings were not adjusted for serologic test accuracy. Conclusions are made on the assumption that most of those infected in the third component of the study (by time of vaccination) were infected with the Delta variant, given its prevalence in Israel.

All data presented are for Maccabi Healthcare Service members. Maccabi members are more likely to come from a higher socioeconomic bracket, and the service has a somewhat larger prevalence of members 35-55 years of age than that for the total population of Israel (17). Although these differences would not affect stratified data in our study, these differences might effect mean serologic results for the total population and vaccine effectiveness results. Generalizability of results to Israel and other countries should be made cautiously.

Given these limitations, the different elements of the study were based on large numbers of a vaccinated population who had 6 months of follow-up time to measure COVID-19 infection. We found that serologic levels for all groups decreased over time and that there was an association between serologic levels and subsequent infection. We further observed that persons vaccinated early in the vaccination campaign had higher infection rates. These factors taken together suggest that the BNT162b2 vaccine, as indicated by the manufacturer, offers lower protection against infection over time, independent of SARS-CoV-2 variant type. These results contributed to the decision to offer a third dose of the BNT162b2 vaccine to persons $\geq 60$ years of age. Follow-up of infection and illness rates in this group will enable us to confirm the wisdom of providing a booster dose. 


\section{About the Author}

Ms. Kertes is a senior researcher in the Division of Data and Digital Health, Maccabi Healthcare Services Tel AvivJaffa, Israel. Her primary research interests are design and implementation of public health studies, public health and health promotion studies (especially regarding smoking), and pharma-therapeutic studies.

\section{References}

1. Rosen B, Waitzberg R, Israeli A. Israel's rapid rollout of vaccinations for COVID-19. Isr J Health Policy Res. 2021;10:6. https://doi.org/10.1186/s13584-021-00440-6

2. Leshem E, Wilder-Smith A. COVID-19 vaccine impact in Israel and a way out of the pandemic. Lancet. 2021;397:17835. https:// doi.org/10.1016/S0140-6736(21)01018-7

3. Vaccines and Related Biological Products Advisory Committee Meeting. December 10, 2020. FDA briefing documentPfizer BioNTech COVID-19 vaccine 2021 [cited 2021 Nov 16]. https://www.fda.gov/media/144245/download

4. Polack FP, Thomas SJ, Kitchin N, Absalon J, Gurtman A, Lockhart S, et al.; C4591001 Clinical Trial Group. Safety and efficacy of the BNT162b2 mRNA COVID-19 vaccine. N Engl J Med. 2020;383:2603-15. https:/ /doi.org/10.1056/ NEJMoa2034577

5. Haas EJ, Angulo FJ, McLaughlin JM, Anis E, Singer SR, Khan F, et al. Impact and effectiveness of mRNA BNT162b2 vaccine against SARS-CoV-2 infections and COVID-19 cases, hospitalisations, and deaths following a nationwide vaccination campaign in Israel: an observational study using national surveillance data. Lancet. 2021;397:1819-29. https://doi.org/10.1016/S0140-6736(21)00947-8

6. Dagan N, Barda N, Kepten E, Miron O, Perchik S, Katz MA, et al. BNT162b2 mRNA COVID-19 vaccine in a nationwide mass vaccination setting. N Engl J Med. 2021;384:1412-23. https:/ / doi.org/10.1056/NEJMoa2101765

7. Thomas SJ, Moreira ED, Kitchin N, Absalon J, Gurtman A, Lockhart S, et al. Six month safety and efficacy of the BNT162b2 mRNA COVID-19 vaccine. N Engl J Med. 2021;385:1761-73.

8. Lopez Bernal J, Andrews N, Gower C, Gallagher E, Simmons R, Thelwall S, et al. Effectiveness of COVID-19 vaccines against the B.1.617.2 (Delta) variant. N Engl J Med. 2021;385:585-94. https://doi.org/10.1056/NEJMoa2108891
9. Zhao J, Yuan Q, Wang H, Liu W, Liao X, Su Y, et al. Antibody responses to SARS-CoV-2 in patients of novel coronavirus disease 2019. Clin Infect Dis. 2020;71:2027-34. https://doi.org/10.1093/cid/ciaa344

10. Zhou W, Xu X, Chang Z, Wang H, Zhong X, Tong X, et al. The dynamic changes of serum IgM and IgG against SARS-CoV-2 in patients with COVID-19. J Med Virol. 2021;93:924-33. https:// doi.org/10.1002/jmv.26353

11. Harvey RA, Rassen JA, Kabelac CA, Turenne W, Leonard S, Klesh R, et al. Association of SARS-CoV-2 seropositive antibody test with risk of future infection. JAMA Intern Med. 2021;181:672-9. https://doi.org/10.1001/ jamainternmed.2021.0366

12. Jalkanen $P$, Kolehmainen $P$, Häkkinen HK, Huttunen $M$, Tähtinen PA, Lundberg R, et al. COVID-19 mRNA vaccine induced antibody responses against three SARS-CoV-2 variants. Nat Commun. 2021;12:3991. https:/ / doi.org/ 10.1038 / s41467-021-24285-4

13. Grupel D, Gazit S, Schreiber L, Nadler V, Wolf T, Lazar R, et al. Kinetics of SARS-CoV-2 anti-S IgG after BNT162b2 vaccination. Vaccine. 2021;39:5337-40. https://doi.org/ 10.1016/j.vaccine.2021.08.025

14. Wei J, Stoesser N, Matthews PC, Ayoubkhani D, Studley R, Bell I, et al.; COVID-19 Infection Survey team. Antibody responses to SARS-CoV-2 vaccines in 45,965 adults from the general population of the United Kingdom. Nat Microbiol. 2021;6:1140-9. https:/ /doi.org/10.1038/ s41564-021-00947-3

15. Cox RJ, Brokstad KA. Not just antibodies: B cells and T cells mediate immunity to COVID-19. Nat Rev Immunol. 2020;20:581-2. https://doi.org/10.1038/s41577-020-00436-4

16. Khoury DS, Cromer D, Reynaldi A, Schlub TE, Wheatley AK, Juno JA, et al. Neutralizing antibody levels are highly predictive of immune protection from symptomatic SARS-CoV-2 infection. Nat Med. 2021;27:1205-11. https://doi.org/10.1038/s41591-021-01377-8

17. Damari N, Cohen R. Health maintenance organizations 2018-2019. Periodic surveys number 317, October 2020. National Insurance Agency, Israel [cited 2021 Nov 16]. https:/ / www.btl.gov.il/Publications/survey/Documents/ seker317/seker_317.pdf

Address for correspondence: Jennifer Kertes, Division of Data and Digital Health, Maccabi Healthcare Services, Rehov HaMered 27, Tel Aviv-Jaffa 16825, Israel, email: dortal_j@mac.org.il 